\title{
Joubert syndrome: genotyping a Northern European patient cohort
}

\author{
Hester Y Kroes ${ }^{\star,}$, Glen R Monroe ${ }^{1}$, Bert van der Zwaag ${ }^{1}$, Karen J Duran ${ }^{1}$, Carolien G de Kovel ${ }^{1}$, \\ Mark J van Roosmalen ${ }^{1}$, Magdalena Harakalova ${ }^{1}$, Ies J Nijman ${ }^{1}$, Wigard P Kloosterman ${ }^{1}$, Rachel H Giles ${ }^{2}$, \\ Nine VAM Knoers ${ }^{1}$ and Gijs van Haaften ${ }^{1}$
}

\begin{abstract}
Joubert syndrome (JBS) is a rare neurodevelopmental disorder belonging to the group of ciliary diseases. JBS is genetically heterogeneous, with $>\mathbf{2 0}$ causative genes identified to date. A molecular diagnosis of JBS is essential for prediction of disease progression and genetic counseling. We developed a targeted next-generation sequencing (NGS) approach for parallel sequencing of 22 known JBS genes plus 599 additional ciliary genes. This method was used to genotype a cohort of 51 well-phenotyped Northern European JBS cases (in some of the cases, Sanger sequencing of individual JBS genes had been performed previously). Altogether, 21 of the 51 cases $(41 \%)$ harbored biallelic pathogenic mutations in known JBS genes, including 14 mutations not previously described. Mutations in C5orf42 (12\%), TMEM67 (10\%), and AHI1 (8\%) were the most prevalent. C5orf42 mutations result in a purely neurological Joubert phenotype, in one case associated with postaxial polydactyly. Our study represents a population-based cohort of JBS patients not enriched for consanguinity, providing insight into the relative importance of the different JBS genes in a Northern European population. Mutations in C5orf42 are relatively frequent (possibly due to a Dutch founder mutation) and mutations in CEP290 are underrepresented compared with international cohorts. Furthermore, we report a case with heterozygous mutations in CC2D2A and B9D1, a gene associated with the more severe Meckel-Gruber syndrome that was recently published as a potential new JBS gene, and discuss the significance of this finding. European Journal of Human Genetics (2016) 24, 214-220; doi:10.1038/ejhg.2015.84; published online 29 April 2015
\end{abstract}

\section{INTRODUCTION}

Joubert syndrome (JBS; OMIM \#213300) is a neurodevelopmental disorder belonging to the group of the ciliopathies, diseases caused by dysfunction of the primary cilia. Like many other ciliopathies, the syndrome encompasses a broad range of clinical symptoms. Its core phenotype is neurological, including intellectual disability, hypotonia, (truncal) ataxia, and oculomotor apraxia. Brain imaging of JBS patients reveals a variable degree of cerebellar vermis hypoplasia and midbrain anomalies, resulting in the characteristic 'molar tooth' midbrain-hindbrain malformation (molar tooth sign (MTS)). The MTS is the key diagnostic feature for this disease. ${ }^{1}$ Extraneurological symptoms in JBS include cystic kidney disease, retinal dystrophy and colobomas, polydactyly, neural tube defects, and liver fibrosis. ${ }^{2,3}$ JBS is in most cases inherited as an autosomal recessive disease, and is extremely clinically and genetically heterogeneous, overlapping with several other ciliopathies. ${ }^{2}$

A clinical diagnosis of JBS can be made by brain imaging showing the MTS in a child with psychomotor retardation. ${ }^{1}$ The prognosis of the disease for an individual patient is difficult to predict: JBS shows a wide range of intellectual disability (from near normal to severe), associated disease (from normal health to life-threatening renal and hepatic dysfunction), and visual involvement (from normal to blindness). ${ }^{3}$ Elucidating the underlying genetic defect may aid in prognosis and in targeted screening for secondary disease as many genotype-phenotype correlations are known for JBS. ${ }^{2,4}$ A molecular diagnosis also allows for accurate genetic counseling and the option of prenatal diagnosis and preimplantation genetic diagnostics.

However, the molecular diagnostics of JBS is challenging, given the fact that $>20$ genes have been identified to date (see OMIM (http://www.ncbi.nlm.nih.gov/omim/).We developed a targeted nextgeneration sequencing (NGS) approach to sequence the 22 known JBS genes and 599 candidate genes selected from the Ciliary Proteome Database $^{5}$ to improve molecular diagnostics for JBS. Given the fact that according to the literature the underlying causative gene is detected in only 40 to $50 \%$ of the cases of JBS, ${ }^{6,7}$ we also investigated candidate genes and the possibility of digenic inheritance that has been suggested in ciliopathies previously. 8,9

Over the years, we collected a unique cohort of 51 Northern European JBS cases, all fulfilling the diagnostic criteria for JBS. Most published studies on JBS are strongly enriched for consanguinity, with an increased ascertainment of cases from populations where consanguinity is common. In our cohort, parental consanguinity was relatively low (five cases $(\sim 10 \%)$, all from Dutch families originating from the Middle East and Northern Africa).

\section{MATERIALS AND METHODS}

Patients

Since 2002, we systematically ascertained JBS patients prospectively and retrospectively from multiple sources, including the Dutch Joubert Syndrome Patient and Parents' Network, Dutch pediatric neurologists, pediatric ophthalmologists, and clinical geneticists. Approval from the National Medical Ethics

${ }^{1}$ Department of Medical Genetics, University Medical Center Utrecht, Utrecht, The Netherlands; ${ }^{2}$ Department of Nephrology and Hypertension, University Medical Center Utrecht, Utrecht, The Netherlands

*Correspondence: HY Kroes, Department of Medical Genetics, University Medical Center Utrecht, Postbox 85090,3508 AB Utrecht, The Netherlands. Tel: +31 887553800 ; Fax: +31 88750 3801; E-mail: h.y.kroes@umcutrecht.nl

Received 23 July 2014; revised 9 February 2015; accepted 25 March 2015; published online 29 April 2015 
Board (CCMO) was obtained before recruitment. We evaluated clinical data and neuroimaging records to check whether the patients met the diagnostic criteria set by Maria et $a l^{10}$ in 1999 or by Saraiva and Baraitser ${ }^{11}$ in 1992 in cases where brain imaging was of insufficient quality to evaluate the MTS (in older cases). A total of 51 cases fulfilled the diagnostic criteria. Most patients had the Dutch nationality, three cases were Flemish and one case was German. In 25 cases, Sanger sequencing of the first gene discovered for JBS, AHI1, was performed previously (see Kroes et $\mathrm{al}^{12}$ ). In two cases, Sanger sequencing in a diagnostic setting of CEP290 and OFD1, respectively, had yielded a molecular diagnosis. We chose to include all cases in order to maintain a full and unbiased cohort for the present study.

\section{A targeted NGS array}

We developed a targeted NGS array containing 621 putative and confirmed ciliary genes, referred to hereafter as the Joubertome. We selected all the known genes involved in ciliopathies at that time, including 22 JBS genes (January 2013; AHI1, CEP290, NPHP1, TMEM216, TMEM67, ARL13B, INPPE5, CC2D2A, OFD1, RPGRIP1L, TTC21B, C5orf42, KIF7, TECT1, CEP41, TMEM237, TMEM138, TMEM231, TCTN2, TCTN3, IFT172, and ZNF423). For the selection of candidate genes we used the Ciliary Proteome Database, containing ciliary proteins assembled from 11 studies (http://v3.ciliaproteome. org). We included all genes from three studies by Andersen et al ${ }^{13}$ (containing centrosomal located genes in human retinal cells), by Blacque et al, ${ }^{14}$ and by Efimenko et $\mathrm{al}^{15}$ (containing C. elegans genes that were selected because of an $\mathrm{x}$-box upstream regulatory motif). ${ }^{13-15}$ Given the fact that there is an excess of male patients in JBS, we also selected all X-linked ciliary genes (45 genes). We added 35 ciliary genes from chromosomal regions identified by homozygosity mapping (Human CNV370-Quadv3 Illumina bead array, Illumina, San Diego, CA, USA) in one consanguineous family. Furthermore, we added candidate genes based on the literature on retinal and renal ciliary genes ${ }^{16}$ (see Supplementary Table S1 for a list of all included genes).

\section{Technical procedures}

Deep sequencing of the Joubertome was performed on two pooled sample cohorts of 32 and 34 cases, of which 51 cases had a diagnosis of JBS (the other 15 samples were from cases with other possible ciliopathies, and are excluded from this study). In all cases array comparative genome hybridization (CGH) had been performed previously. This did not yield copy number variants (CNVs) in regions with JBS-associated genes (including the large deletion on chromosome 2q13 encompassing the NPHP1 gene that has been reported a number of times in JBS patients ${ }^{2}$ ).

A total of 60-nucleotide-long probes uniquely mapped to coding sequences of all genes from the GRCh37/hg19 human reference genome (http://www. ncbi.nlm.nih.gov/assembly/2758) with $50 \mathrm{bp}$ flanks into intronic regions were designed with an average tiling density of $4 \mathrm{bp}$ on average for both positive and negative strands. The size of the targeted region was $2.7 \mathrm{Mb}$, covered by 779592 probes. Fragment library preparation and genomic enrichment on a $1 \mathrm{M}$ custom microarray (Agilent Technologies, Santa Clara, CA, USA) was performed as previously described. ${ }^{17}$ Briefly, $2 \mu \mathrm{g}$ of purified gDNA (Qiagen, Hilden, Germany) was sheared into $150 \mathrm{bp}$ fragments with a Covaris S2 sonicator (Covaris, Woburn, MA, USA), then blunt-ended and 5'-phosphorylated, and finally shortened double-stranded adaptors complementary with the SOLiD next-generation sequencing platform were ligated to the ends. Next, the nonphosphorylated and nonligated $3^{\prime}$ ends were nick-translated, and bar-coded sequences unique to each sample were added using seven PCR cycles. Amplified library fragments were pooled into two pools of 32 and 34 samples, respectively, and a region of $150-225 \mathrm{bp}$ was excised from a $2 \%$ agarose gel. Following gel purification (Qiagen), samples were enriched using a custom protocol ${ }^{17}$ and amplified in 13 PCR cycles with primers complementary to the full SOLiD oligo sequences. The enriched pool was then used for emulsion PCR following the manufacturer's instructions (Life Technologies, Carlsbad, CA, USA). The pooled samples were run as a full slide on the SOLiD 5500XL. Following SOLiD sequencing, color space reads were mapped against GRCH37/hg19 reference genome using a custom pipeline based on the BWA software (http://bio-bwa.sourceforge.net), and variants and small indels were annotated as described previously. ${ }^{17}$ Average sample coverage was 147X and 136X, and 92 and $89 \%$ of requested sequences were covered by $>20$ reads for run 1 and 2 respectively. Run 2 had a higher fraction of duplicated reads compared with run 1, probably generated during PCR amplification steps post enrichment.

\section{Bioinformatic analysis}

The criteria for variant detection coverage were set at 10 reads, and a nonstringent cutoff for heterozygote allele calls was set at $25-75 \%$. Common alleles were filtered from rare or novel variants using the reference frequencies from the data sets of NCBI dbSNP Build 137 for Human (https://www.ncbi. nlm.nih.gov/SNP), Exome Variant Server (EVS) (http://evs.gs.washington.edu/ EVS), 1000Genomes (http://www.1000genomes.org), Genome of the Netherlands (http://www.nlgenome.nl), and our in-house data set containing exome data of over 100 individuals to exclude platform-based errors. The conservation score and predicted effect on protein function were calculated using prediction programs (GERP, PolyPhen2, SIFT, Provean) for variant prioritization. Confirmation of the selected SNVs and segregation analysis in the family were performed by Sanger sequencing. Primer information is available upon request. The variants identified in this study were submitted to the Leiden Open Variant Database (www.LOVD.nl, patient IDs 28912-28926, 28947-28952).

\section{RESULTS}

\section{Mutation detection}

In Table 1 , the cases in which a molecular diagnosis was made are summarized. In 21 out of 51 cases (41\%), homozygous/compound heterozygous mutations in known JBS genes were present. Of these 38 mutations, 14 mutations were novel.

Homozygous/compound heterozygous mutations in C5orf 42 were detected in 6 cases $(\sim 12 \%)$, in TMEM67 in 5 cases $(\sim 10 \%)$, and in AHI1 in 4 cases $(\sim 8 \%)$. One of these cases, born of consanguineous parents, had a homozygous TMEM67 missense mutation and a homozygous missense mutation in AHI1, both predicted to be damaging. The symptoms in this patient are consistent with a TMEM67-associated JBS phenotype (colobomatous microphthalmia, elevated liver enzymes).

In addition, homozygous/compound heterozygous mutations were present in INPP5E ( 2 cases, 4\%), RPGRIP1L (1 case, 2\%), TMEM231 (1 case, 2\%), CC2D2A (1 case, 2\%), and CEP290 ( 1 case, $2 \%$ ), and hemizygous in the X-linked JBS gene OFD1 (1 case, $2 \%)$. No biallelic mutations were found in NPHP1, TMEM216, ARL13B, TTC21B, KIF7, CEP41, TMEM237, TMEM138, TCTN1, TCTN2, TCTN3, IFT172, and ZNF423.

The cohort contained five cases with previously known mutations in JBS genes. Of these nine disease-causing alleles, we identified five in our study. The four mutations we missed were all small insertions or deletions, (Table 1, mutations printed in italic). These are known to be harder to identify using NGS technology.

In one case (not mentioned in the table) we found a mosaic hemizygous one-base pair deletion in OFD1, c.710delA, p.(Lys237Serfs ${ }^{\star} 6$ ). In a control experiment, this mutation was found back in low-grade mosaicism in normal controls as well (see also Piton et al). ${ }^{18}$ The deletion is probably a PCR-induced artifact in this mononucleotide repeat.

In Supplementary Table S2, all mutations that were detected in known JBS genes in the entire patient cohort are shown. In 16 out of the 30 cases $(53 \%)$ without a molecular diagnosis, one heterozygous mutation predicted 'probably damaging' or 'deleterious' by SIFT/Polyphen was present.

Case 1.37 revealed a heterozygous damaging missense mutation in $C C 2 D 2 A$, and also two mutations in a possible novel gene for JBS, B9D1. ${ }^{19}$ The B9D1 mutations were c.151T >C p.Ser51Pro, predicted to be damaging, and $17: 19246826 \mathrm{C}>\mathrm{A}$, that leads to an 


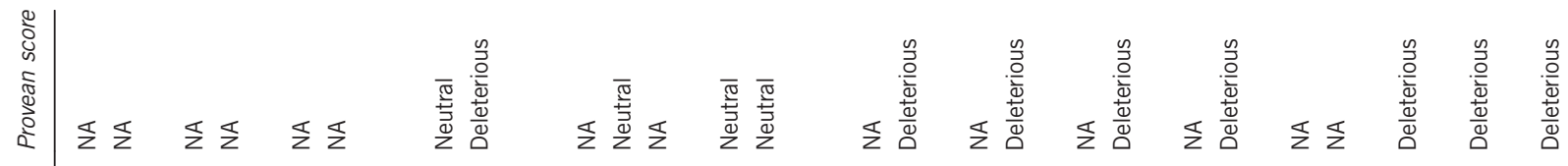

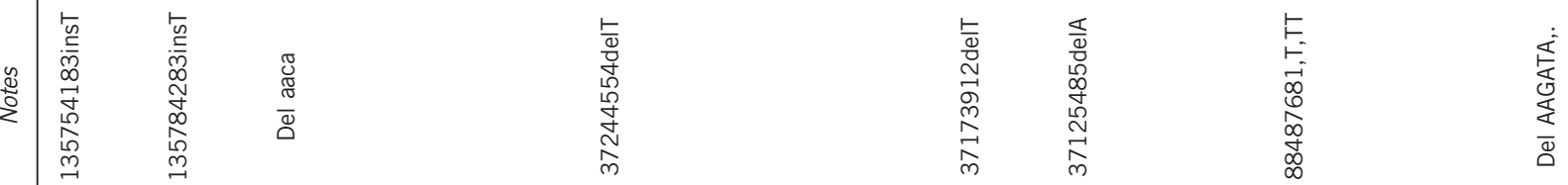

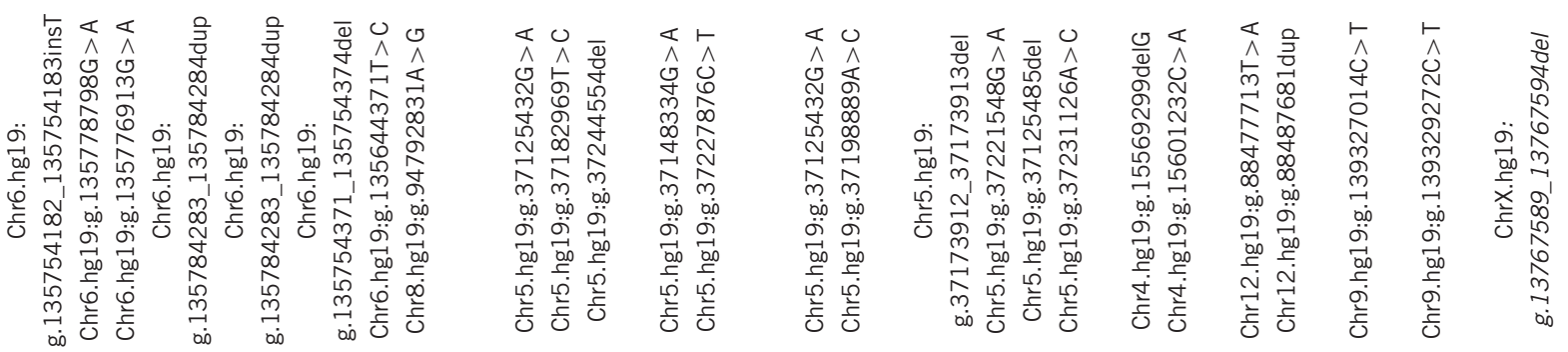

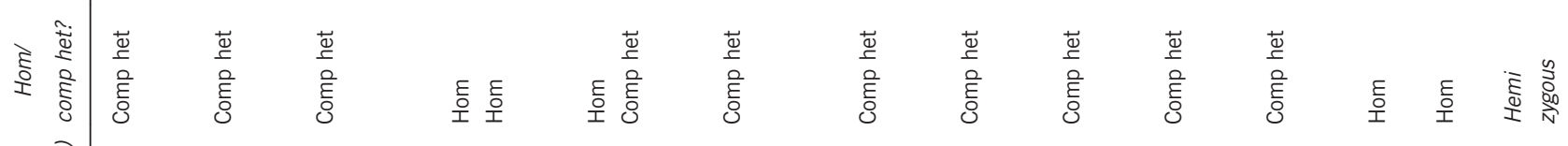

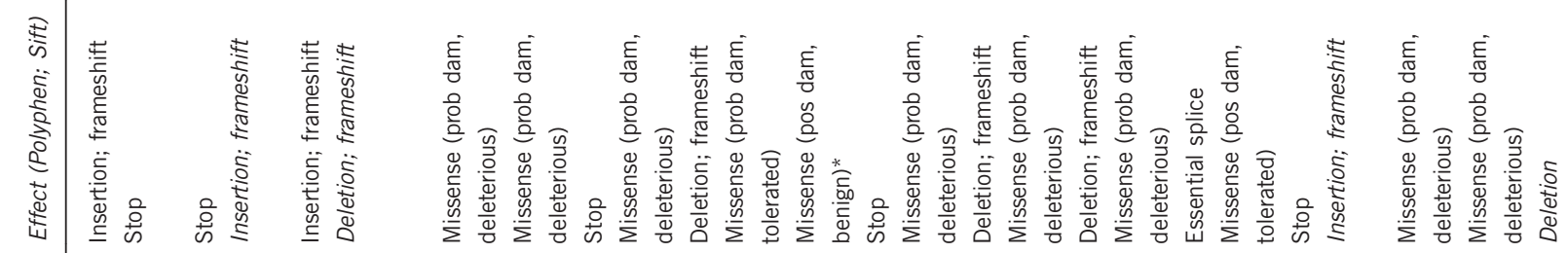

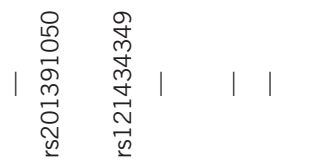

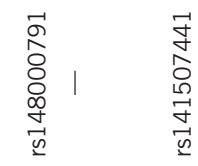

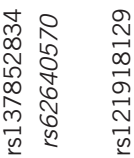

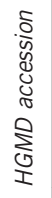

(1)

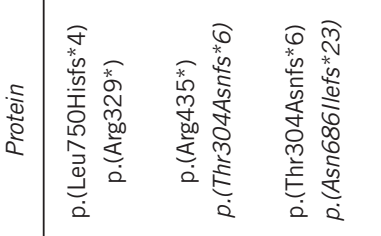

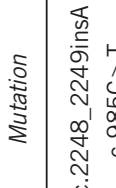
 范 美

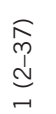

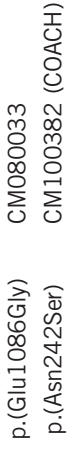

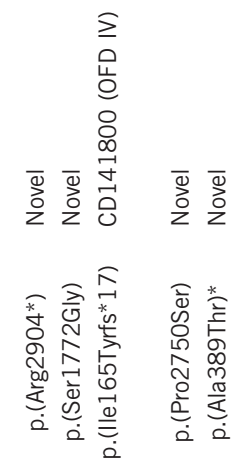

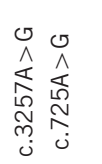

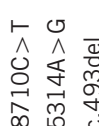

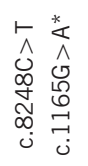

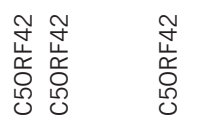

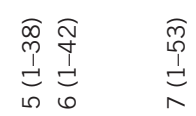

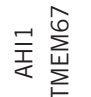

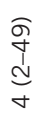

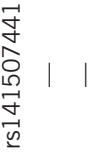

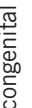<smiles>[CH]1[CH]C[CH]1</smiles>

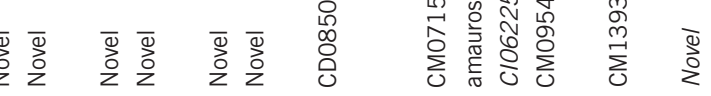

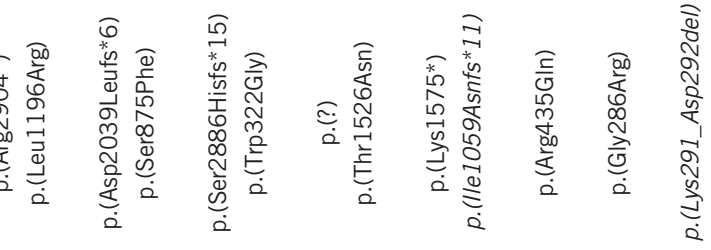

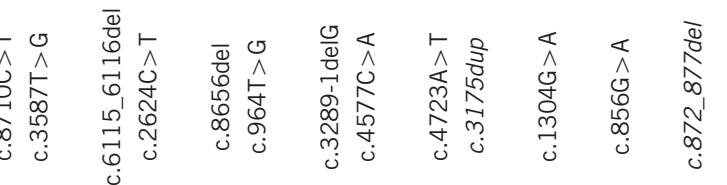

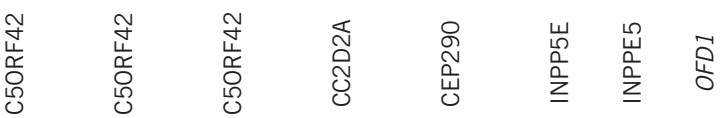

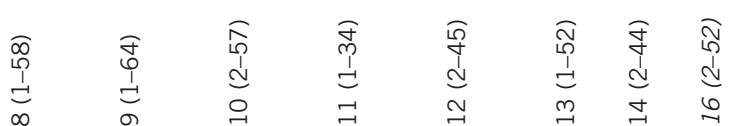




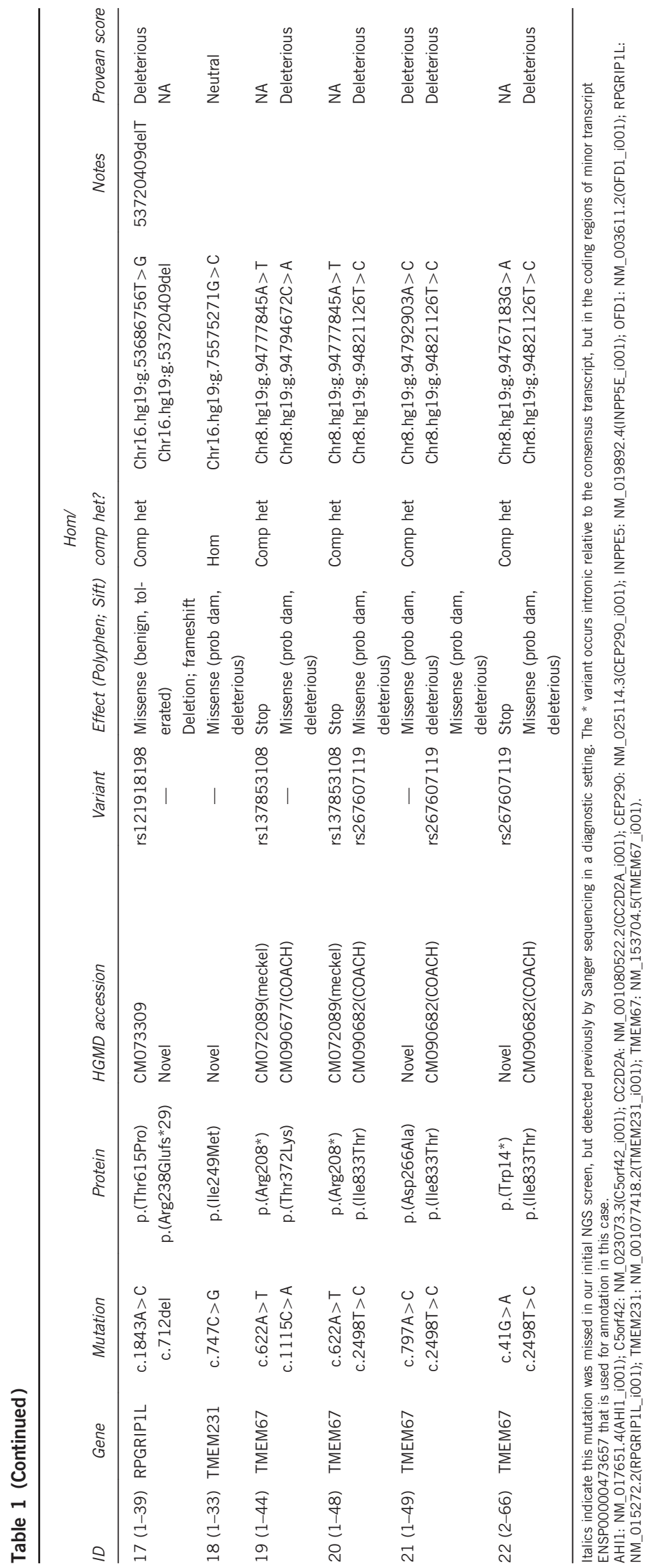


amino-acid change p.Trp170Cys in one alternative B9D1 transcript (ENST00000395616), and is predicted to be benign.

Biallelic deleterious mutations were detected in two candidate genes that are currently under investigation. No deleterious mutations in $\mathrm{X}$-linked candidate genes were detected, despite an excess of male patients in our JBS cohort $(\mathrm{M} / \mathrm{F}=35: 16)$ and in the literature (see, eg, Saraiva and Baraitser ${ }^{11}$ and Chance et $\left.a^{20}\right)$.

\section{Genotype-phenotype correlations}

The clinical details of JBS cases with a genetic diagnosis are summarized in Table 2. Of the six patients with mutations in C5orf42, five show a purely neurological phenotype, including oculomotor apraxia and/or typical breathing pattern (age range 2-37 years), and one case also showed a postaxial polydactyly of four limbs (age 32 years). One of the two cases with INPP5E mutations had endstage renal insufficiency. Cystic kidney disease was present in only 2 of the 31 INPP5E-associated JBS cases with full clinical details reported in the literature. ${ }^{21,22}$ Combining these data, the chance of cystic kidney disease in INPP5E-related JBS seems at least 10\%. All other cases fit and consolidate the phenotypes that are proposed in the literature, ${ }^{2,4}$ although some patients do not show the full clinical spectrum (possibly because of their young age).

\section{DISCUSSION}

C5orf42, TMEM67, and AHI1 are important JBS genes in the Northern European population

The genes C5orf42, TMEM67, and AHI1 are the most frequently mutated genes in this Northern European cohort (in respectively 12, 10 , and $8 \%$ of cases). In C5orf 42 , the c.8710C $>\mathrm{T}$ mutation could represent a 'Dutch' founder mutation. Haplotype analysis of this allele indeed showed convincing linkage disequilibrium in line with shared ancestry (see Supplementary Data S1). Comparing our data with those of international cohorts reported in the literature (see Supplementary Table S3), the contribution of C5orf42 seems relatively high. ${ }^{2,6}$ The contribution of CEP290, on the contrary, is low (2 versus $8-10 \%$ ). We checked whether JBS patients with CEP290 mutations were ascertained elsewhere in the Netherlands, but this was not the case. Taken together, it would seem that the Dutch population tends to have a relatively high contribution of C5orf42, possibly because of a Dutch founder mutation, and a low contribution of CEP290. CSPP1, anticipated to be mutated in $2-5 \%$ of JBS cases, ${ }^{6,23}$ was not analyzed in our cohort and may yet resolve a few additional cases.

C5orf42 is associated with 'classic' JBS, with or without polydactyly C5orf42 is a recently discovered JBS gene, and the clinical spectrum of C5orf42 mutations in JBS is slowly emerging. In our cohort, five out of the six patients with C5orf 42 mutations show a purely neurological phenotype. Intellectual impairment seems mild relative to other JBS patients (the three older patients can all read and write, one patient lives semi-independently). All patients are physically healthy, without kidney or liver disease. This is similar to the cases described in the literature. $^{24-26}$ One of our cases showed polydactyly of all limbs that was postaxial in all instances (in the literature, preaxial and mesoaxial polydactyly is also reported frequently). ${ }^{24,26,27}$ In another case reported in the literature (from a consanguineous mating), an occipital meningocele and abnormal electroretinogram (ERG) were described, ${ }^{25}$ neither of which were present in our cases.

Oral-facial-digital syndrome type VI (OFD VI) is regarded as one of the rare phenotypic subtypes of JBS. ${ }^{28}$ There has been some debate in the literature whether C5orf 42 may be the major gene for OFD VI. ${ }^{26,27}$ None of the six cases in our cohort showed typical signs of OFD VI, whereas no deleterious variants were found in any of the genes tested in the one case in our cohort with the OFD VI phenotype.

One of our cases (1.42) carries the same heterozygous frameshift mutation as two OFD VI cases (c.493delA p.Ile165Tyrfs ${ }^{\star} 17$ ). The most likely explanation for this phenotypical variation seems to be the involvement of modifier genes.

In conclusion, the combined data of this study and the literature show that C5orf 42 mutations in most cases lead to the classic form of JBS, sometimes associated with polydactyly but without evidence for cystic kidney disease, liver fibrosis, or retinal dystrophy. In a small subset of cases, C5orf42 mutations may result in JBS type OFD VI.

\section{Non-Mendelian inheritance and B9D1}

Digenic inheritance in JBS was suggested by Lee et al ${ }^{8}$ in 2012, who identified CEP41 as a new autosomal recessive JBS gene, but also reported three cases with a heterozygous CEP41 mutation combined with a heterozygous mutation in KIF7 or CC2D2A. We found no convincing proof for digenic inheritance involving known JBS genes. Three cases had heterozygous missense mutations in two JBS genes (case 1.43: CEP290 and RGRIP1L; case 1.62: KIF7 and CEP290; and case 2.47: C5orf42 and CC2D2A ); however, at least one of the two mutations in every case may not be pathogenic according to functional prediction programs (see Supplementary Table S2). We searched the literature (PubMed), public protein interaction databases (BioGRID, HPRD, IntAct), and public functional interaction network tools (GeneMANIA, STRING) for experimental proof of functional interactions between the mutated JBS genes in these three cases, but did not find any evidence for specific interactions.

In one case (1.37) we detected a heterozygous missense mutation in $C C 2 D 2 A$ combined with a heterozygous mutation that were both predicted deleterious in B9D1, a gene associated with Meckel-Gruber syndrome. ${ }^{29,30}$ This case also had a second missense mutation in B9D1 resulting in an amino-acid change predicted to be benign in one transcript and situated in the $3^{\prime}$ UTR of most other transcripts. The function of this alternative transcript or impact on $3^{\prime}$ UTR functioning is unknown at the moment. Parental DNA was unavailable to confirm that the mutations were on different alleles. B9D1 and CC2D2A colocalize at the transition zone of the cilium and belong to a conglomerate of proteins called the B9 complex. ${ }^{31-34}$ Several other JBS proteins are also predicted to be part of this complex. These simultaneous variants in $C C 2 D 2 A$ and $B 9 D 1$ may suggest a digenic or triallelic inheritance model given the functional interaction between the two proteins. Alternatively, we could have missed a mutation on the second $C C 2 D 2 A$ allele, or this case may represent a third example of $B 9 D 1$ as a novel JBS gene. ${ }^{19}$ This would be in line with the observation that mild mutations in MKS-associated genes can cause JBS, for example, RPGRIP1L/MKS5 and CC2D2A/MKS6. ${ }^{35,36}$ The patient presented with a mild classic JBS phenotype without renal, retinal, or hepatic disease (at age 10 years), similar to the phenotype of two JBS cases with B9D1 mutations described recently. ${ }^{19}$

The percentage of JBS cases with a heterozygous mutation in a known JBS gene $(53 \%)$ is much higher than expected in a rare autosomal recessive disease (the estimated birth prevalence of JBS is 1 in 80000 to 1 in 100000); 2,7 however, we cannot exclude that a second pathogenic mutation is present but missed by our detection method. Theoretically, these mutations could act as modifier genes, or fit into a digenic or oligogenic inheritance model.

\section{Proposed molecular diagnostic strategy for JBS}

NGS technology is particularly useful in a heterogeneous disease like JBS. We chose a targeted approach of all known JBS genes as an initial 


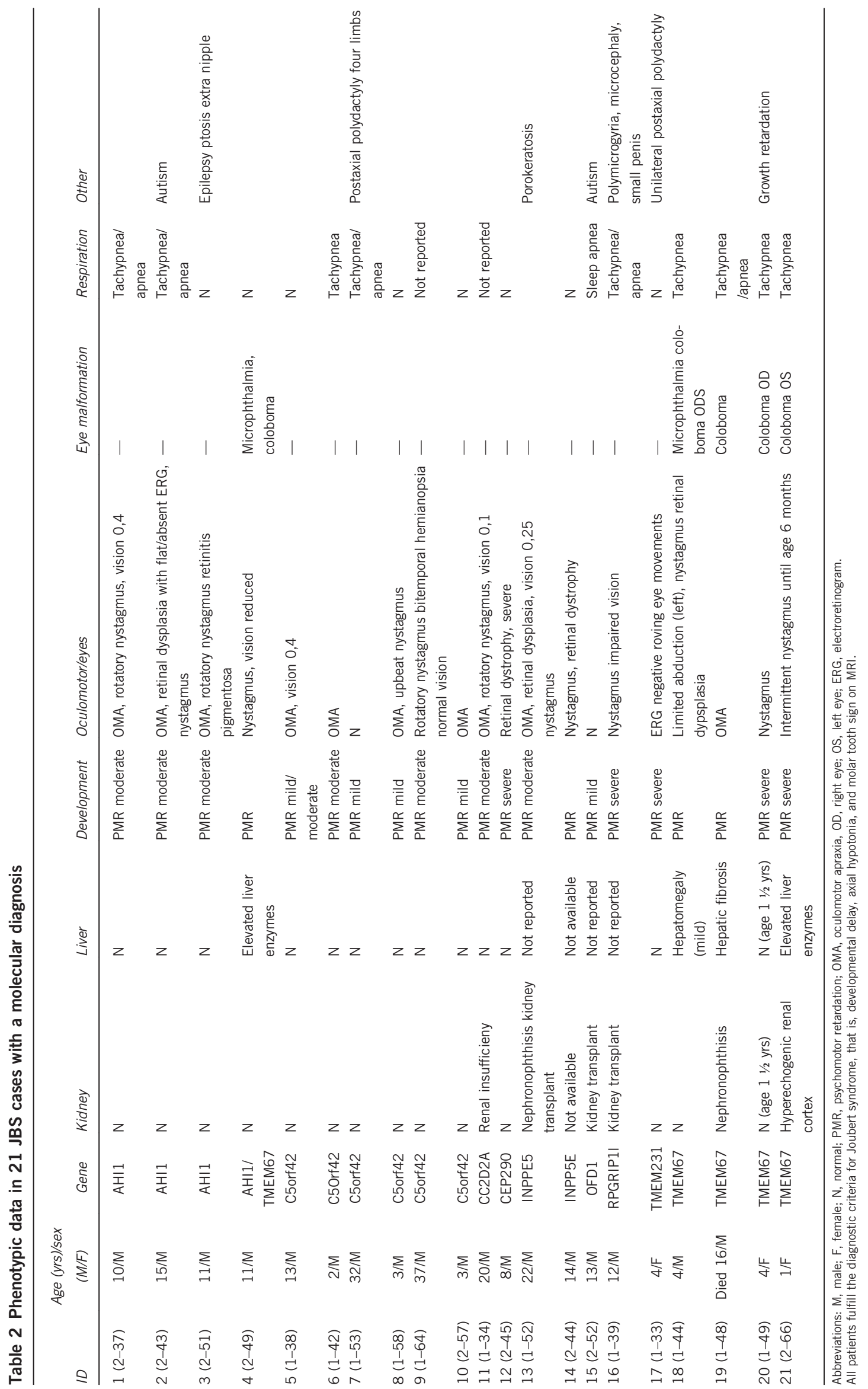


step, given the allelic heterogeneity of $\mathrm{JBS}^{2}$ that forecludes a reliable prediction of the genotype based on the phenotype. This study shows that detection of small insertions and deletions may prove challenging by the NGS technology employed here, given the false negative rate of four out of nine mutations, all four small insertions/deletions that were detected earlier by Sanger sequencing. We improved on this by switching from array-based to in-solution enrichment resulting in a higher read depth, and adjusting the bioinformatics pipeline. Validation of these adjustments in a diagnostic setting proved that indels $<6$ bps can be detected now with a success rate of $\sim 80 \%$.

As current capture technologies fail to enrich the full coding region, there is an additional role for Sanger sequencing. In cases heterozygous for a mutation in a known JBS gene, Sanger sequencing and investigation of larger deletions (by MLPA for instance) is the preferred next step. Given the large size of many of the known JBS genes, however, this may not always be feasible in a diagnostic setting.

This two-step approach of a NGS gene panel followed by prioritized Sanger sequencing is time efficient against overall lower cost compared with sequential Sanger sequencing of individual genes. Over the next few years, WES will offer an opportunity to detect so far elusive novel JBS genes. The rarity of the mutations in these novel genes stresses the importance of pooling of data and collaboration between the groups worldwide involved in JBS and other ciliopathies. Specific attention should be paid to interactions of genes that belong to known functional pathways within the cilium, keeping an open mind to non-Mendelian inheritance patterns.

\section{CONFLICT OF INTEREST}

The authors declare no conflict of interest.

\section{ACKNOWLEDGEMENTS}

We thank all the children and their parents who have participated in this study for their cooperation and willingness to share their experiences with us. This research was supported by grants from the Dutch Kidney Foundation (CP11.18 to NVAMK and RHG).

1 Maria BL, Quisling RG, Rosainz LC et al: Molar tooth sign in Joubert syndrome: clinical, radiologic, and pathologic significance. J Child Neurol 1999; 14: 368-376.

2 Parisi M, Glass I: Joubert Syndrome and related disorders. Gene Rev 1993, rev. 2013.

3 Doherty D: Joubert syndrome: insights into brain development, cilium biology, and complex disease. Semin Pediatr Neurol 2009; 16: 143-154.

4 Valente EM, Brancati F, Dallapiccola B: Genotypes and phenotypes of Joubert syndrome and related disorders. Eur J Med Genet 2008; 51: 1-23.

5 Gherman A, Davis EE, Katsanis N: The ciliary proteome database: an integrated community resource for the genetic and functional dissection of cilia. Nat Genet 2006; 38: 961-962.

6 Akizu N, Silhavy JL, Rosti RO et al: Mutations in CSPP1 lead to classical Joubert syndrome. Am J Hum Genet 2014; 94: 80-86.

7 Valente EM, Brancati F, Boltshauser E, Dallapiccola B: Clinical utility gene card for: Joubert syndrome-update 2013. Eur J Hum Genet 2013; 21.

8 Lee JE, Silhavy JL, Zaki MS et al: CEP41 is mutated in Joubert syndrome and is required for tubulin glutamylation at the cilium. Nat Genet 2012; 44: 193-199.

9 Schaffer AA: Digenic inheritance in medical genetics. J Med Genet 2013; 50: 641-652.
10 Maria BL, Boltshauser E, Palmer SC, Tran TX: Clinical features and revised diagnostic criteria in Joubert syndrome. J Child Neurol 1999; 14: 583-590.

11 Saraiva JM, Baraitser M: Joubert syndrome: a review. Am J Med Genet 1992; 43: 726-731.

12 Kroes HY, van Zon PH, Fransen van de Putte D et al: DNA analysis of AHI1, NPHP1 and CYCLIN D1 in Joubert syndrome patients from the Netherlands. Eur J Med Genet 2008; 5: 24-34.

13 Andersen JS, Wilkinson CJ, Mayor T, Mortensen P, Nigg EA, Mann M: Proteomic characterization of the human centrosome by protein correlation profiling. Nature 2003; 426: 570-574.

14 Blacque OE, Perens EA, Boroevich KA et al: Functional genomics of the cilium, a sensory organelle. Curr Biol 2005; 15: 935-941.

15 Efimenko E, Bubb K, Mak HY et al: Analysis of xbx genes in C. elegans. Development 2005; 132: 1923-1934.

16 Otto EA, Hurd TW, Airik R et al: Candidate exome capture identifies mutation of SDCCAG8 as the cause of a retinal-renal ciliopathy. Nat Genet 2010; 42: 840-850.

17 Harakalova M, Mokry M, Hrdlickova B et al: Multiplexed array-based and in-solution genomic enrichment for flexible and cost-effective targeted next-generation sequencing. Nat Protoc 2011; 6: 1870-1886.

18 Piton A, Redin C, Mandel JL: XLID-causing mutations and associated genes challenged in light of data from large-scale human exome sequencing. Am J Hum Genet 2013; 93: 368-383.

19 Romani M, Micalizzi A, Kraoua I et al: Mutations in B9D1 and MKS1 cause mild Joubert syndrome: expanding the genetic overlap with the lethal ciliopathy Meckel syndrome. Orphanet J Rare Dis 2014; 9: 72.

20 Chance PF, Cavalier L, Satran D, Pellegrino JE, Koenig M, Dobyns WB: Clinical nosologic and genetic aspects of Joubert and related syndromes. J Child Neurol 1999; 14: 660-666.

21 Bielas SL, Silhavy JL, Brancati F et al: Mutations in INPP5E, encoding inositol polyphosphate-5-phosphatase $\mathrm{E}$, link phosphatidyl inositol signaling to the ciliopathies. Nat Genet 2009; 41: 1032-1036.

22 Travaglini L, Brancati F, Silhavy J et al: Phenotypic spectrum and prevalence of INPP5E mutations in Joubert syndrome and related disorders. Eur J Hum Genet 2013; 21: 1074-1078.

23 Tuz K, Bachmann-Gagescu R, O'Day DR et al: Mutations in CSPP1 cause primary cilia abnormalities and Joubert syndrome with or without Jeune asphyxiating thoracic dystrophy. Am J Hum Genet 2014; 94: 62-72.

24 Srour M, Schwartzentruber J, Hamdan FF et al: Mutations in C5orf42 cause Joubert syndrome in the French Canadian population. Am J Hum Genet 2012; 90: 693-700.

25 Alazami AM, Alshammari MJ, Salih MA et al: Molecular characterization of Joubert syndrome in Saudi Arabia. Hum Mutat 2012; 33: 1423-1428.

26 Romani M, Mancini F, Micalizzi A et al: Oral-facial-digital syndrome type VI: is C5orf42 really the major gene? Hum Genet 2015; 134: 123-126.

27 Lopez E, Thauvin-Robinet C, Reversade B et al: C5orf42 is the major gene responsible for OFD syndrome type VI. Hum Genet 2014; 133: 367-377.

28 Poretti A, Vitiello G, Hennekam RC et al: Delineation and diagnostic criteria of oralfacial-digital syndrome type VI. Orphanet J Rare Dis 2012; 7: 4.

29 Hopp K, Heyer CM, Hommerding CJ et al: B9D1 is revealed as a novel Meckel syndrome (MKS) gene by targeted exon-enriched next-generation sequencing and deletion analysis. Hum Mol Genet 2011; 20: 2524-2534.

30 Dowdle WE, Robinson JF, Kneist A et al: Disruption of a ciliary B9 protein complex causes Meckel syndrome. Am J Hum Genet 2011; 89: 94-110.

31 Garcia-Gonzalo FR, Corbit KC, Sirerol-Piquer MS et al: A transition zone complex regulates mammalian ciliogenesis and ciliary membrane composition. Nat Genet 2011; 43: 776-784.

32 Chih B, Liu P, Chinn Y et al: A ciliopathy complex at the transition zone protect the cilia as privileged membrane domain. Nat Cell Biol 2012; 14: 61-72.

33 Sang L, Miller JJ, Corbit KC et al: Mapping the NPHP-JBTS-MKS protein network reveals ciliopathy disease genes and pathways. Cell 2011; 145: 513-528.

34 Gate D, Danielpour M, Levy R, Breunig JJ, Town T: Basic biology and mechanisms of neural ciliogenesis and the B9 family. Mol Neurobiol 2012; 45: 564-570.

35 Mougou-Zerelli S, Thomas S, Szenker E et al: CC2D2A mutations in Meckel and Joubert syndromes indicate a genotype-phenotype correlation. Hum Mutat 2009; 30: 1574-1582.

36 Delous M, Baala L, Salomon R et al: The ciliary gene RPGRIP1L is mutated in cerebello-oculo-renal syndrome (Joubert syndrome type B) and Meckel syndrome. Nat Genet 2007; 39: 875-881.

Supplementary Information accompanies this paper on European Journal of Human Genetics website (http://www.nature.com/ejhg) 